\title{
Accelerated DNA methylation changes in middle-aged men define sexual dimorphism in human lifespans
}

\author{
Fu-Hui Xiao 1,2,3,4, Xiao-Qiong Chen 1,2,3,4, Yong-Han He 1,2,3,4 and Qing-Peng Kong 1,2,3,4*
}

\begin{abstract}
Background: Accelerated age-associated DNA methylation changes in males may explain the earlier onset of agerelated diseases (e.g., cardiovascular disease (CVD)) and thus contribute to sexually dimorphic morbidity and lifespan. However, the details regarding the emergence of this sex-biased methylation pattern remain unclear.

Results: To address these issues, we collected publicly available peripheral blood methylation datasets detected by Illumina HumanMethylation450 BeadChip platform from four studies that contain age and gender information of samples. We analyzed peripheral blood methylation data screened from 708 subjects of European ancestry. Results revealed a significant methylation change acceleration in middle-aged males (40-50 years old), which was further supported by another cohort containing 2711 subjects with Indian ancestry. Additional analyses suggested that these sexually dimorphic methylation changes were significantly overrepresented in genes associated with CVD, which may impact the potential activation of disease expression. Furthermore, we showed that higher prevalence of drinking and smoking in the males has some contribution to the sex-based methylation patterns during aging.

Conclusion: Our results indicated that the sex-biased methylation changes occurred in middle-aged men in an acceleration manner and likely contribute to the sexual dimorphism observed in human lifespan by promoting the occurrence of CVD. As drinking and smoking were also found to be associated with this accelerated methylation change in men, it is possible that male lifespan may be prolonged by improving unhealthy lifestyles at or before middle age.
\end{abstract}

Keywords: DNA methylation, Middle-age, Cardiovascular disease, Sexual dimorphism, Lifespan

\section{Introduction}

It is well known that men often live shorter than women [1], which is partly explained by their higher mortality and earlier onset of some age-related diseases, especially cardiovascular disease (CVD) $[2,3]$. Accumulated evidence showed that epigenetic changes in aging process are associated with lifespan and age-related diseases including CVD [4-7], indicating the roles of epigenetic mechanisms in sexually dimorphic lifespan. As the well-known epigenetic modification, DNA methylation

\footnotetext{
* Correspondence: kongqp@mail.kiz.ac.cn

'State Key Laboratory of Genetic Resources and Evolution/Key Laboratory of Healthy Aging Research of Yunnan Province, Kunming Institute of Zoology, Chinese Academy of Sciences, Kunming 650223, China

${ }^{2}$ Center for Excellence in Animal Evolution and Genetics, Chinese Academy of Sciences, Kunming 650223, China

Full list of author information is available at the end of the article
}

plays an important role in the regulation of gene transcription [8]. Sexually divergent DNA methylation changes during aging have been observed in animals and humans $[9,10]$. Notably, a previous study showed that diverse age-related DNA methylation changes have some associations with CVD incidence difference in crowds [11]. Especially, men do possess an accelerated methylation change during aging, which is supposed to contribute to the earlier onset of CVD and shorter lifespan [11, 12]. However, the ways or causes for this sexually dimorphic methylation change pattern remain largely unclear. In this study, we have therefore analyzed the sexual differences of methylation profiles (Illumina HumanMethylation450 BeadChip) during aging in two independent cohorts containing 708 and 2711 samples [12-15], respectively, and explored the contribution of some risk factors (i.e., drinking and smoking) to these sexual methylation differences. 


\section{Results and discussion}

By analyzing the methylation profile data (covering over 485,000 CpG sites) from 708 healthy individuals (of European ancestry), screened by the Illumina HumanMethylation450 BeadChip (Additional file 1: Table S1) [12-14], we identified 357 CpGs showing significant sexually dimorphic changes in methylation (see the "Materials and Methods" section). Interestingly, changes in most of these CpGs (290/ 357 ) were accelerated in men (Fig. 1a and Additional file 1: Table S2). We annotated the distribution of the above 290 CpGs and found they were located on 185 genes, the overrepresentation of which are associated with CVD (Fig. 1b and Additional file 1: Table S2). For example, cg14519515 locates in the promoter of $A D R B K 1$, a gene with elevated expression in CVD patients [16, 17]. The decreased methylation level of this $\mathrm{CpG}$ site during aging likely upregulates the expression of $A D R B K 1$ in men and contributes to the earlier onset of CVD (Additional file 1: Figure S1A, B). An additional case comes from cg20222376. This CpG site locates in the promoter of $A K A P 8 L$ and displays reduced methylation level with age (Additional file 1: Figure S1C).
The methylation level of cg20222376 is positively associated with the expression level of AKAP8L (Additional file 1: Figure S1D). Given the high expression of AKAPs can protect the cardiovascular system [18] and men contain a lower methylation level at this CpG locus compared with age-matched women, it is likely that the methylation pattern will increase the risk of CVD in men. Similar to observations reported in previous research [11], our results suggested that these sex-biased methylation changes may play a role in promoting the occurrence of CVD in men. Coincidently, we also observed that methylation changes were accelerated in males and occurred 6.9 years earlier, on average, than the changes in females (Fig. 1c), echoing the observation that CVD develops 7-10 years earlier in men [3].

We next explored how these sexually dimorphic methylation differences emerge during aging. The methylation differences in the 290 identified CpGs were compared among different age groups, as shown in Fig. 1d and Additional file 1: Figure S2A, B. Surprisingly, a significantly accelerated sex-based methylation difference occurred in the 40-50-year-old age group, in sharp

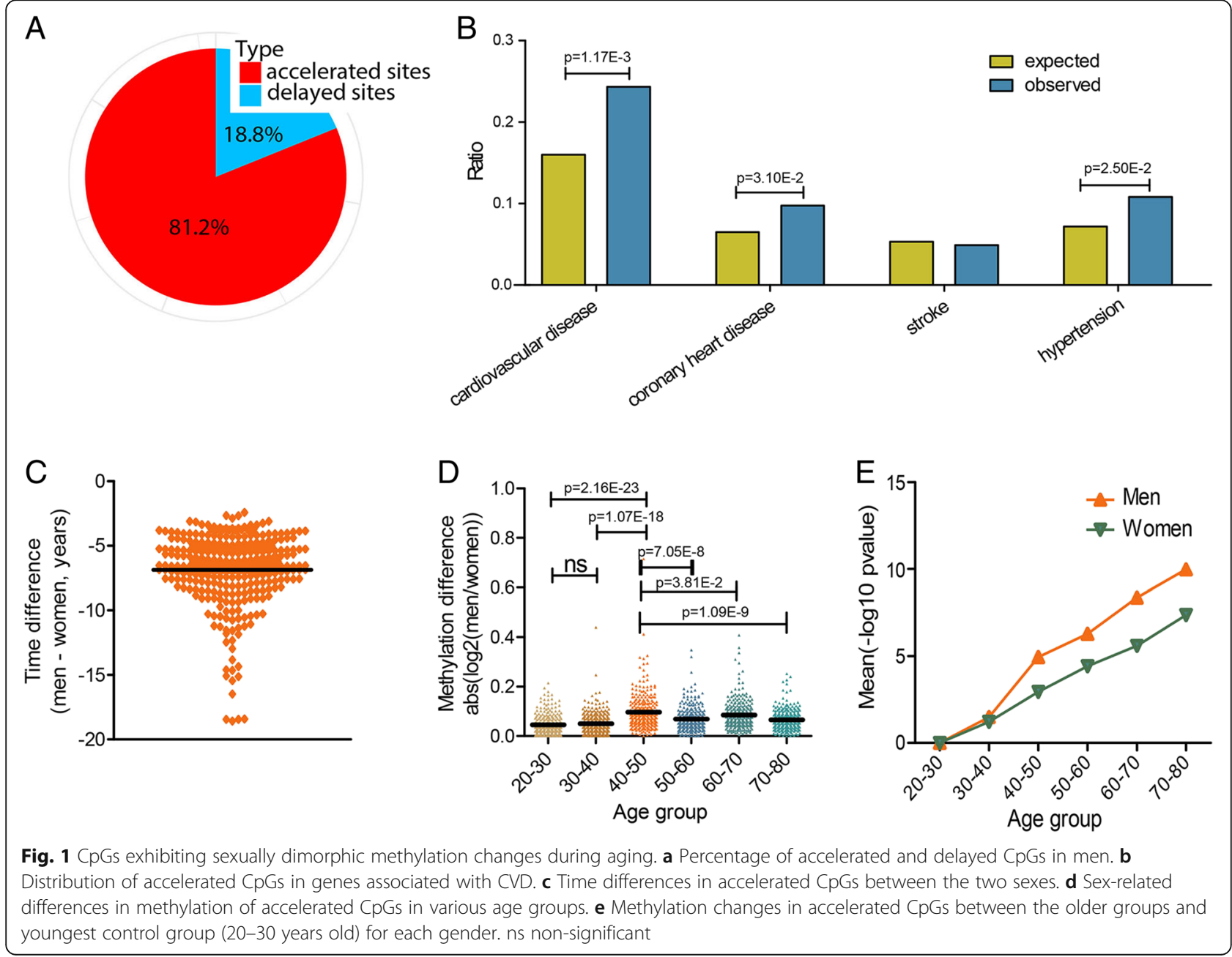


contrast with the observation that the degree of difference in methylation between the two sexes was relatively small before the age of 40 . Consistently, age-related analysis in each gender revealed an abruptly accelerated methylation change in middle-aged men (Fig. 1e).

To determine the factors associated with the accelerated methylation change in middle-aged males, we analyzed the influence of drinking and smoking, two well-known risk factors with higher prevalence in men that contribute, at least in part, to their high risk of CVD and shorter lifespan [19-24]. By analyzing available public methylation dataset with drinking information [25], we found that, among the 290 identified CpGs, 70 showed methylation differences between drinkers and non-drinkers $(p<0.05)$. All of the $70 \mathrm{CpGs}$ exhibited the same directional changes in drinkers as those with age (Fig. 2a). A similar pattern was observed in smokers by the methylation dataset collected in females (the males being unavailable) [26]. Sixty-seven of the 290 CpGs were differentially methylated in smokers compared with non-smokers $(p<0.05)$, with most of them CpGs $(51 / 67)$ showing the same directional changes in smokers as those with age (Fig. 2b).

To test whether our observations were confined to a certain population or could be observed in others, we analyzed another methylation dataset from London Life Sciences Prospective Population (LOLIPOP) study containing 2711 subjects with Indian ancestry (Additional file 1: Table S1) [15]. We identified $709 \mathrm{CpGs}$ that exhibited sex-based differences in methylation during aging, with most (596/709) showing significant acceleration in men (on average, 5.4 years earlier in males than females; Fig. 3a and Additional file 1: Table S3). Again, sharply accelerated methylation changes were observed in middle-aged men (Fig. 3b, c and Additional file 1: Figure S3A, B). Importantly, the $596 \mathrm{CpGs}$ were again overrepresented in genes associated with CVD (Fig. 3d and Additional file 1: Table S3). Moreover, 66 and 180 of the CpGs were differentially methylated in drinkers and smokers, respectively $(p<0.05)$, with most $(59 / 66,162 / 180)$ exhibiting identical directional changes as those with age (Fig. 3e, f).

A previous study has suggested that the rapid DNA methylation changes during aging in men contribute to the earlier onset of CVD and shorter lifespan [11]. Our study further identified that such acceleration emerges in middle-aged men, with drinking and smoking found to be associated with these changes, and thus likely has some contribution to the acceleration. This observation is in accordance with epidemiological findings of abruptly increased CVD mortality in men aged 4554 years old [2]. Since we have observed the same pattern in the two cohorts with different ancestries and thus most plausibly distinct genetic backgrounds, it is then unlikely that our observation is race specific. Our study therefore raises the possibility that controlling these aberrant epigenetic modifications at or before middle age via lifestyle changes, e.g., smoking and alcohol reduction or abstinence, could help reduce the incidence of CVD in men and thus prolong their lifespans.

\section{Materials and methods \\ Data collection}

The methylation data, generated by the Illumina HumanMethylation450 BeadChip (HM450), were collected from NCBI's Gene Expression Omnibus (GEO) datasets. One integrated dataset was downloaded with accession numbers GSE32148, GSE41169, and GSE40279, in which only data from healthy samples were considered (Additional file 1: Table S1) [12-14]; another dataset was downloaded with the accession number GSE55763 (Additional file 1: Table S1) [15]. Two additional datasets containing smoking and drinking information were downloaded with accession numbers
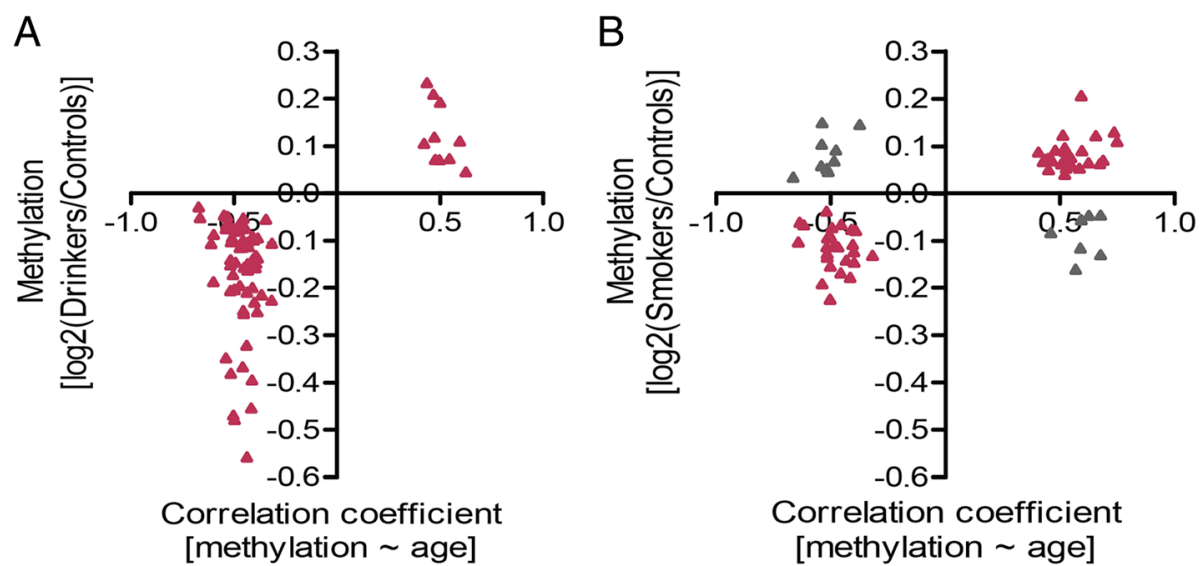

Fig. 2 Effects of drinking and smoking on sex-related differences in methylation changes. a Accelerated CpGs differentially methylated in drinkers. b Accelerated CpGs differentially methylated in smokers 


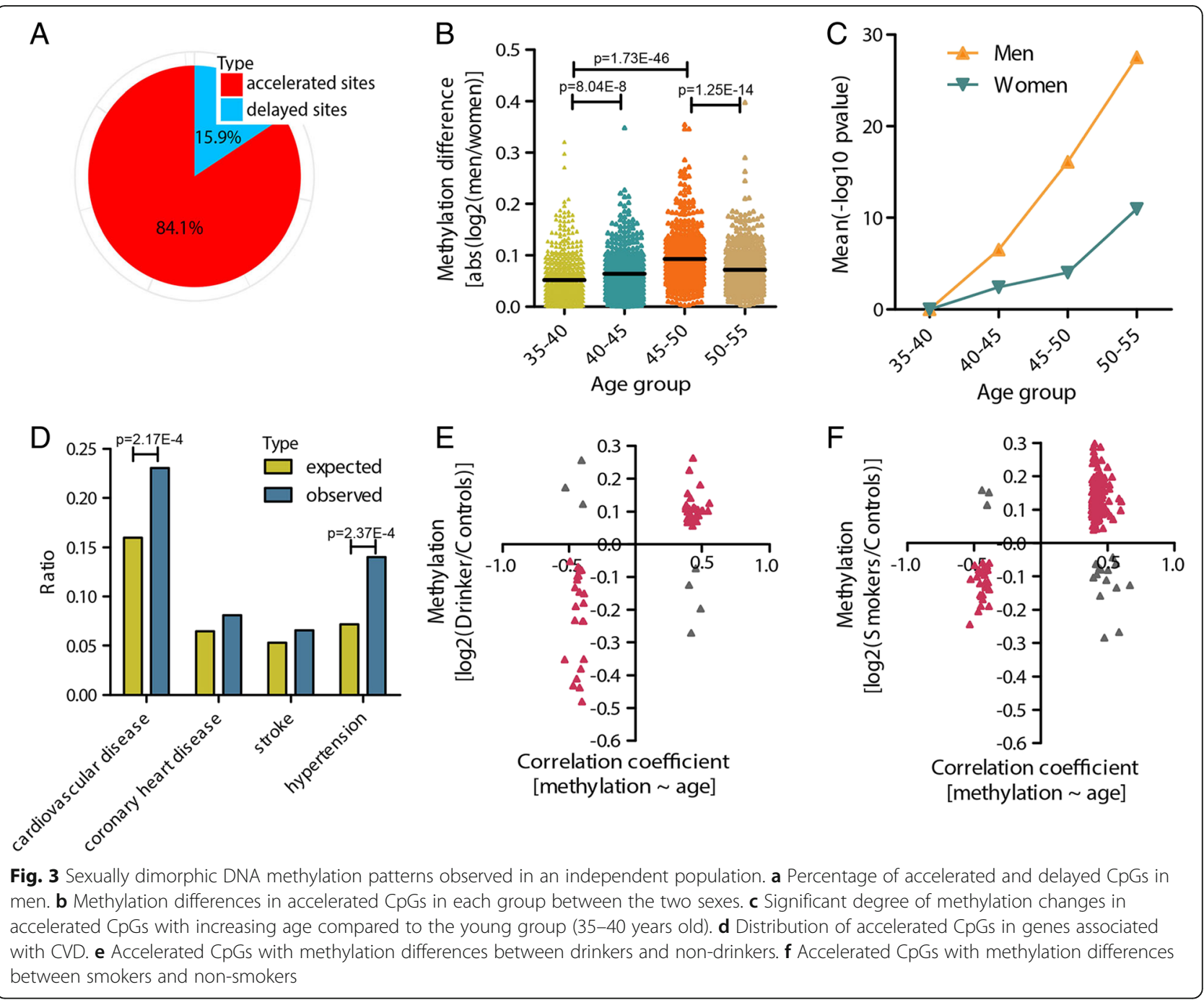

GSE53045 and GSE57853 [25, 26]. The methylation level of each CpG site is represented by a beta value (range $0-1$ ). The 364 normal tissue samples with RNA-seq (version 2) and methylation (HM450) data were downloaded from The Cancer Genome Atlas (TCGA) Data Portal website (https:// tcga-data.nci.nih.gov/). Any sites with missing values in more than $5 \%$ of samples were discarded, and the remaining CpG sites with missing values in a few subjects were filled with the $\mathrm{R}$ "impute" package [27]. The values were then normalized using quantile normalization with the normalize.quantiles function in the $\mathrm{R}$ "preprocessCore" package (http://www.bioconductor.org/).

\section{Identification of $\mathrm{CpGs}$ with sexually dimorphic methylation changes}

We first calculated the coefficient of variance (cv) of all CpG sites and filtered out those with values less than the interquartile range (IQR) cut-off of 0.5. A stepwise polynomial regression model $\left(\operatorname{step}\left(\operatorname{lm}\left(y \sim 1+A g e+I\left(A g e^{\wedge} 2\right)+\right.\right.\right.$ $\left.\left.I\left(A g e^{\wedge} 3\right)\right)\right)$, direction = "backward") was then used to find the best-fit model. Sites showing significantly age-related changes were identified using the $F$ test between the best-fit and null models $(y \sim 1)$. The CpG sites with $p$ values $[F$ test, Benjamini and Hochberg $(\mathrm{BH})$ corrected] $<0.01$ and adjusted $R^{2}>0.25$ were retained for further analysis. To improve the credibility of the above sites, a nonparametric Spearman rank correlation test was adopted, and only the sites with absolute correlation coefficient values of $>0.2$ were regarded as age-related CpG sites. We then used multiple linear regression with the best-fit model to identify the CpG sites with sex-related differences. To test the heterochrony (age shift) for the sex-biased CpG sites, we defined the age-related methylation change curve of men as the reference object for each CpG site and used a nonlinear least-squares algorithm (NL2SOL) to find the optimal age shift between the two sexes. The significance of the age transformation was assessed using the $F$ test, as described in detail elsewhere [28]. Here, the age shift for each CpG was calculated, in which "age-shift $<0$ " represents the 
curve of men located on the left side of women and thus defined as "accelerated sites in men", otherwise, on the right side.

\section{Gene annotation, enrichment, and correlation analyses} Gene information on the $\mathrm{CpG}$ sites of interest was obtained from the "IlluminaHumanMethylation450k.db" database. The genes associated with age-related diseases were collected from GeneCards version 3.12 by searching the names including cardiovascular disease, coronary heart disease, stroke, and hypertension [29]. A hypergeometric test was performed to find the enriched disease terms based on the observed and expected gene numbers. A Pearson correlation test was performed to test the relationship between the methylation of each $\mathrm{CpG}$ site and the expression of its corresponding gene with the data from TCGA, including the 364 normal tissue samples with both RNA-seq and HM450 data (https://tcga-data.nci.nih.gov/, see the "Data collection" section).

\section{Analysis of sex-related methylation change patterns}

The mean methylation differences in one age group between the two sexes for each $\mathrm{CpG}$ were calculated and used to compare with adjacent age groups. We also evaluated the significant degree of methylation changes with increasing age for each gender by comparing the older and youngest groups (control). Statistical significance was calculated with Student's $t$ tests.

\section{Identification of differentially methylated $\mathrm{CpGs}$ in smokers or drinkers}

Using the methylation datasets with drinking and smoking information (see the "Data collection" section), we analyzed the CpGs with methylation differences between the two groups (smokers/drinkers versus controls) using Student's $t$ tests.

\section{Additional file}

\section{Additional file 1: Figure S1. Illustrations for the roles of the sexually} dimorphic methylation in promoting occurrence of cardiovascular disease in men. Figure S2. Methylation differences of the identified 290 CpGs (with accelerated methylation changes in males) between the two sexes among different age groups. Figure S3. Sexually dimorphic methylation in the CpGs with accelerated methylation changes in males. Table S1. Summary of the collected DNA methylation datasets. Table S2. Information on the 290 accelerated CpG sites in the first dataset. Table S3. Information on the 574 accelerated CpG sites in the second dataset. (DOC 1996 kb)

\section{Abbreviations}

ADRBK1: Adrenergic beta receptor kinase 1; AKAP8L: A-kinase-anchoring protein 8-like; BH: Benjamini and Hochberg; cv: Coefficient of variance; CVD: Cardiovascular disease; HM450: Illumina HumanMethylation450 BeadChip; IQR: Interquartile range; NL2SOL: Nonlinear least-squares algorithm

\section{Funding}

This study was supported by grants from the National Natural Science Foundation of China (91749109, 81701394, 81671404, and 81500670), Applied Basic Research Projects of Yunnan Province (2017FA038, 2018FB121), Chinese Academy of Sciences (QYZDB-SSW-SMC020), Youth Innovation Promotion Association of the Chinese Academy of Sciences (to Q.P.K and Y.H.H.), and CAS "Light of West China" Program (to F.H.X.).

\section{Availability of data and materials}

The datasets collected and analyzed in this study are available in the Gene Expression Omnibus repository (GEO) ( https://www.ncbi.nlm.nih.gov/geo/) and The Cancer Genome Atlas (TCGA) Data Portal (https://tcga-data.nci.nih.gov/) (see the "Data collection" section in "Materials and Methods").

\section{Authors' contributions}

QPK designed and supervised the project. FHX performed data analyses. FHX performed literature research. FHX and QPK wrote the paper. XQC and YHH revised the manuscript. All authors read and approved the final manuscript.

\section{Ethics approval and consent to participate}

This study was approved by the Ethics Committee at Kunming Institute of Zoology, Chinese Academy of Sciences.

\section{Consent for publication}

Not applicable.

\section{Competing interests}

The authors declare that they have no competing interests.

\section{Publisher's Note}

Springer Nature remains neutral with regard to jurisdictional claims in published maps and institutional affiliations.

\section{Author details}

${ }^{1}$ State Key Laboratory of Genetic Resources and Evolution/Key Laboratory of Healthy Aging Research of Yunnan Province, Kunming Institute of Zoology, Chinese Academy of Sciences, Kunming 650223, China. ${ }^{2}$ Center for Excellence in Animal Evolution and Genetics, Chinese Academy of Sciences, Kunming 650223, China. ${ }^{3}$ Kunming Key Laboratory of Healthy Aging Study, Kunming 650223, China. ${ }^{4}$ KIZ/CUHK Joint Laboratory of Bioresources and Molecular Research in Common Diseases, Kunming 650223, China.

Received: 1 August 2018 Accepted: 21 October 2018

Published online: 29 October 2018

References

1. Robine JM, Romieu I, Cambois E. Health expectancy indicators. B World Health Organ. 1999:77(2):181-5

2. Lerner DJ, Kannel WB. Patterns of coronary heart disease morbidity and mortality in the sexes: a 26-year follow-up of the Framingham population. Am Heart J. 1986;111(2):383-90.

3. Maas AH, Appelman YE. Gender differences in coronary heart disease. Neth Heart J. 2010;18(12):598-602

4. Calvanese V, Lara E, Kahn A, Fraga MF. The role of epigenetics in aging and age-related diseases. Ageing Res Rev. 2009;8(4):268-76.

5. Xiao $\mathrm{FH}$, Kong QP, Perry $\mathrm{B}, \mathrm{He} \mathrm{YH}$. Progress on the role of DNA methylation in aging and longevity. Brief Funct Genomics. 2016;15(6):454-9.

6. Levine ME, Lu AT, Quach A, Chen BH, Assimes TL, Bandinelli S, Hou L, Baccarelli AA, Stewart JD, Li Y, et al. An epigenetic biomarker of aging for lifespan and healthspan. Aging (Albany NY). 2018;10(4):573-91.

7. Xiao FH, He YH, Li QG, Wu H, Luo LH, Kong QP. A genome-wide scan reveals important roles of DNA methylation in human longevity by regulating age-related disease genes. PLoS One. 2015;10(3):e0120388.

8. Lim D, Maher ER. DNA methylation: a form of epigenetic control of gene expression. Obstet Gynaecol. 2010;12(1):37-42.

9. Masser DR, Hadad N, Porter HL, Mangold CA, Unnikrishnan A, Ford MM, Giles CB, Georgescu C, Dozmorov MG, Wren JD, et al. Sexually divergent DNA methylation patterns with hippocampal aging. Aging Cell. 2017;16(6): 1342-52.

10. Numata $S$, Ye T, Hyde TM, Guitart-Navarro X, Tao R, Wininger M, Colantuoni C, Weinberger DR, Kleinman JE, Lipska BK. DNA methylation signatures in 
development and aging of the human prefrontal cortex. Am J Hum Genet. 2012;90(2):260-72.

11. Horvath S, Gurven M, Levine ME, Trumble BC, Kaplan H, Allayee H, Ritz BR, Chen B, Lu AT, Rickabaugh TM, et al. An epigenetic clock analysis of race/ ethnicity, sex, and coronary heart disease. Genome Biol. 2016;17(1):171.

12. Hannum G, Guinney J, Zhao L, Zhang L, Hughes G, Sadda S, Klotzle B, Bibikova M, Fan JB, Gao Y, et al. Genome-wide methylation profiles reveal quantitative views of human aging rates. Mol Cell. 2013;49(2):359-67.

13. Harris RA, Nagyszakal D, Pedersen N, Opekun AR, Bronsky J, Munkholm P, Jespersgaard C, Andersen PS, Melegh B, Ferry GD. Genome-wide peripheral blood leukocyte DNA methylation microarrays identified a single association with inflammatory bowel diseases. Inflamm Bowel Dis. 2012;18(12):2334-41.

14. Horvath $S$, Zhang $Y$, Langfelder P, Kahn RS, Boks MP, van Eijk K, van den Berg LH, Ophoff RA. Aging effects on DNA methylation modules in human brain and blood tissue. Genome Biol. 2012:13(10):R97.

15. Lehne B, Drong AW, Loh M, Zhang WH, Scott WR, Tan ST, Afzal U, Schulz R, Scott J, Jarvelin MR, et al. A coherent approach for analysis of the Illumina HumanMethylation450 BeadChip improves data quality and performance in epigenome-wide association studies. Genome Biol. 2015;16(1):37.

16. Liu SL, Premont RT, Kontos CD, Zhu SK, Rockey DC. A crucial role for GRK2 in regulation of endothelial cell nitric oxide synthase function in portal hypertension. Nat Med. 2005;11(9):952-8.

17. Lymperopoulos A, Rengo G, Funakoshi H, Eckhart AD, Koch WJ. Adrenal GRK2 upregulation mediates sympathetic overdrive in heart failure. Nat Med. 2007;13(3):315-23.

18. Mauban JR, O'Donnell M, Warrier S, Manni S, Bond M. AKAP-scaffolding proteins and regulation of cardiac physiology. Physiology (Bethesda). 2009; 24:78-87.

19. Burns DM. Epidemiology of smoking-induced cardiovascular disease. Prog Cardiovasc Dis. 2003;46(1):11-29.

20. Emberson JR, Shaper AG, Wannamethee SG, Morris RW, Whincup PH. Alcohol intake in middle age and risk of cardiovascular disease and mortality: accounting for intake variation over time. Am J Epidemiol. 2005; 161(9):856-63.

21. Makela P. Alcohol-related mortality by age and sex and its impact on life expectancy - estimates based on the Finnish death register. Eur J Pub Health. 1998:8(1):43-51.

22. Rehm J, Sulkowska U, Manczuk M, Boffetta P, Powles J, Popova S, Zatonski W. Alcohol accounts for a high proportion of premature mortality in central and eastern Europe. Int J Epidemiol. 2007;36(2):458-67.

23. Valkonen $T$, VanPoppel $F$. The contribution of smoking to sex differences in life expectancy - four Nordic countries and the Netherlands 1970-1989. Eur J Pub Health. 1997;7(3):302-10.

24. Wood AM, Kaptoge S, Butterworth AS. Risk thresholds for alcohol consumption: combined analysis of individual-participant data for 599912 current drinkers in 83 prospective studies. Lancet. 2018:391(10136):2212.

25. Philibert RA, Penaluna B, White T, Shires S, Gunter T, Liesveld J, Erwin C, Hollenbeck N, Osborn T. A pilot examination of the genome-wide DNA methylation signatures of subjects entering and exiting short-term alcohol dependence treatment programs. Epigenetics-Us. 2014:9(9):1212-9.

26. Dogan MV, Shields B, Cutrona C, Gao L, Gibbons FX, Simons R, Monick M, Brody GH, Tan K, Beach SRH, et al. The effect of smoking on DNA methylation of peripheral blood mononuclear cells from African American women. BMC Genomics. 2014;15(1):151.

27. Troyanskaya O, Cantor M, Sherlock G, Brown P, Hastie T, Tibshirani R, Botstein D, Altman RB. Missing value estimation methods for DNA microarrays. Bioinformatics. 2001;17(6):520-5.

28. Somel M, Franz H, Yan Z, Lorenc A, Guo S, Giger T, Kelso J, Nickel B, Dannemann M, Bahn S, et al. Transcriptional neoteny in the human brain. P Natl Acad Sci USA. 2009;106(14):5743-8.

29. Safran M, Dalah I, Alexander J, Rosen N, Iny Stein T, Shmoish M, Nativ N, Bahir I, Doniger T, Krug H, et al. GeneCards Version 3: the human gene integrator. Database (Oxford). 2010;2010:baq020.

Ready to submit your research? Choose BMC and benefit from:

- fast, convenient online submission

- thorough peer review by experienced researchers in your field

- rapid publication on acceptance

- support for research data, including large and complex data types

- gold Open Access which fosters wider collaboration and increased citations

- maximum visibility for your research: over $100 \mathrm{M}$ website views per year

At BMC, research is always in progress.

Learn more biomedcentral.com/submissions 